Disclosure of Interest: H. Maki: None declared, M. Hatano Grant/research support from: Ministry of Health, Labour and Welfare, Bayer Japan, Nippon Shinyaku Co,.Ltd, GlaxoSmithKline K.K., Speakers bureau: Actelion pharmaceuticals Japan Ltd, Bayer Japan, S. Minatsuki: None declared, T. Inaba: None declared, I. Komuro Grant/research support from: Actelion pharmaceuticals Japan Ltd, Y. Asano Grant/research support from: Ministry of Health, Labour and Welfare, S. Sato Grant/research support from: Ministry of Health, Labour and Welfare DOI: 10.1136/annrheumdis-2017-eular.4271

\section{AB0636 A COMPARISON STUDY OF PREVALENCE OF TRADITIONAL CARDIOVASCULAR RISK FACTORS AND FRAMINGHAM RISK SCORE IN SYSTEMIC SCLEROSIS PATIENTS AND MATCHED CONTROLS}

J. Sousa-Neves, M. Cerqueira, D. Santos-Faria, J. Leite Silva, C. Afonso, F. Teixeira. Rheumatology, Hospital Conde de Bertiandos, ULSAM, Ponte de Lima, Portugal

Background: In Systemic Sclerosis (SSc), data on prevalence of traditional cardiovascular (CV) disease risk factors is scarce and conflicting (1). Therefore, SSc patients $\mathrm{CV}$ risk attributed to traditional $\mathrm{CV}$ risk factors remains an issue of debate.

Objectives: To evaluate if patients with SSc have a higher prevalence of traditional $\mathrm{CV}$ disease risk factors and a higher risk of long-term CV events based on the risk prediction tool of the Framingham risk score (FRS) in comparison with age, race and sex matched control subjects.

Methods: The study comprised patients diagnosed with SSc, fulfilling both the $1980 \mathrm{ACR}$ and the 2013 ACR/EULAR criteria for the disease, and followed-up at our Rheumatology Department and a group of age, race and sex-matched controls. Inclusion criteria were age 30 to 74 and no history of CV events in order to calculate FRS. In total, 46 out of 62 patients were eligible for the study. Traditional CV disease risk factors (diabetes, arterial hypertension and smoking) were compared among the 46 patients with SSc and 51 matched controls. Systolic blood pressure (SBP) values and total and high-density lipoprotein (HDL) cholesterol levels were also collected. The 10-year risk for CV events according to FRS was calculated and means of patients and controls were compared. Subjects' distribution into 3 categories of risk - low ( $<10 \%$ risk), medium (10-20\% risk) and high $(>20 \%$ risk) was also compared. Parametric and nonparametric tests were used for comparison between groups. $P$ value $<0.05$ was defined as statistically significant. Results: Mean risk for CV events in 10-years assessed by FRS was $10.00 \% \pm 8.61$ for SSc patients and $7.76 \% \pm 8.30$ for matched controls. Differences were not statistically significant $(p=0.196)$. Additionally, prevalence of diabetes, arterial hypertension and smoking did not differ significantly between the two groups ( $p=0.890, p=0.443, p=0.651$, respectively). Total and HDL cholesterol levels were also similar between groups $(p=0.963$ and $p=0.506$, respectively). Only SBP values $(\mathrm{mmHg})$ of SSc patients were significantly higher $(128.50 \mathrm{mmHg}[113.5$ to 139.3$]$ ) (median [interquartile range]) compared with controls (120.00 [110 to $130]), p=0.031$. Subjects' distribution into the 3 groups of risk defined was similar for both groups $(p=0.205)$.

Conclusions: In our study, prevalence of traditional CV disease risk factors and 10-year risk for CV events based on FRS assessment tool did not differ significantly between SSc patients and age, sex and race matched controls.

\section{References:}

[1] Psarras A, Soulaidopoulos S, Garyfallos A, Kitas G and Dimitroulas T. A critical view on cardiovascular risk in systemic sclerosis. Rheumatol Int. 2017 Jan;37(1):85-95.

Disclosure of Interest: None declared

DOI: 10.1136/annrheumdis-2017-eular.4528

\section{AB0637 SUBCLINICAL ATHEROMATOSIS AND VITAMIN D DEFICIENCY IN PATIENTS WITH SCLERODERMA}

J.J. Gonzalez Martin ${ }^{1}$, O. Carrion ${ }^{2}$, A. Abdelkader ${ }^{2}$, E. Calvo ${ }^{1}$, F. Aramburu ${ }^{1}$, F. Sainz ${ }^{2}$, P. García de la Peña ${ }^{1} .{ }^{1}$ Rheumatology; ${ }^{2}$ Angiology and Vascular Surgery, Hospital Universitario HM Sanchinarro, Madrid, Spain

\section{Objectives:}

To study whether patients with systemic sclerosis (SSc) have an increased cardiovascular risk (CVR), measured on the basis of analytical, angiodinamic and/or vascular lesions on carotid ultrasound.

The carotid IMT is a marker of cardiovascular morbidity and mortality, allowing measurement and monitoring of atherosclerosis in asymptomatic individuals, being surrogate markers of future coronary disease, stroke and general death in the general population and in inflammatory rheumatologic diseases.

Methods: Epidemiological and analytical data were collected, including the determination of the RCV SCORE index.

Vascular ultrasound protocol included assessment of carotid intima-media thickness (IMT), presence of atheromatous plaques, and exploration of peripheral arteriopathy using the ankle arm index (ABI).

Results: Seventy adult patients with ES diagnosis (ACR-EULAT 2013 criteria) were included.

$94 \%$ of the women had a mean age of $50.2 \pm 12.5$ years, and an average evolution time of $3.0 \pm 4.4$ years.
The distribution by subgroups was: limited SSc (48\%), diffuse SSc (34\%), pre-SSc $\left(4^{\prime} 2 \%\right)$, sine SSc $(2.8 \%)$, MCTD ( $57 \%$ ) and overlap syndrome $\left.4^{\prime} 2 \%\right)$. The mean SSRm was $9.3 \pm 7.0$ (range $0-42$ ).

The ANA were positive in $91.4 \%$, ACA $(51.4 \%)$, ATA (10\%), RNA polymerase $(42 \%)$.

$4 \%$ were DM, $7 \%$ were obese, $11 \%$ were active smokers, $13 \%$ were $\mathrm{HTN}$, and $28 \%$ were ex-smokers.

$28 \%$ had hypercholesterolemia with a mean total cholesterol of 192.5 (SD \pm 31.9 ) and LDL of $102.4(S D \pm 29.4 \mathrm{mg} / \mathrm{dL})$.

$57 \%$ received vasodilators, most of them ARA-II. $10 \%$ bosentan, $4.2 \%$ sildenafil, and a $2.8 \%$ combination therapy.

The percentage of immunosuppressive drugs was corticoid (50\%), MTX (34\%), mycophenolate (3\%), AZA (11\%), HCQ (14\%), CP \%).

The IMT presented pathological values $(>0.9 \mathrm{~mm})$ in $39 \%$ of the sample, $23 \%$ had atheroma plaques (being bilateral in $40 \%$ ). Subclinical atheromatosis affected $41.4 \%$ (patients without cardiovascular events, pathological IMT and/or atheroma plaques). The ABI had pathological values $(<0.9)$ in $17 \%$ of the patients.

In the bivariate analysis, the pathological GIM was related to the presence of ACA antibodies (OR $=3.80,95 \% \mathrm{Cl}: 1.15-12.52, \mathrm{p}=0.028)$ and with the SCORE index of CVR (OR $=2.93,95 \% \mathrm{Cl}: 1.12-7,64, \mathrm{p}=0.028)$; And the presence of atherosclerotic plaques was associated with increased SSRm score (OR 1.09, 95\% $\mathrm{Cl} 1.00-1.19, \mathrm{p}=0.046$ ), and the highest CVR SCORE index (OR 3.90, 95\% $\mathrm{Cl}: 1.31-11.56, p=0.014$.

In the multivariate analysis, the serum vitamin $\mathrm{D}$ concentration showed a protective effect on IMT (OR $=0.94,95 \% \mathrm{Cl} 0.89-0.99$, $\mathrm{p}$ value $=0.025)$; And the main determinant of atheromatous plaques is the SCORE index, since the increase of one unit in SCORE index multiplies by 4 the probability of presenting plaques (OR $=4.06,95 \% \mathrm{Cl}: 1.31-12.60 ; \mathrm{P}=0.015)$, once the effect of SSRm was controlled.

Conclusions:

- $40 \%$ of the patients had pathological IMT values, showing association with the presence of positive AAC and the SCORE risk index.

- The serum concentration of $25-\mathrm{OH}$-vitamin $\mathrm{D}$ showed a protective effect on IMT. Sixty percent of the sample had vitamin $D$ deficiency.

- The presence of atheromatous plaques ( $23 \%$ of patients) was associated with higher SSRm indexes and SCORE cardiovascular risk.

Disclosure of Interest: None declared

DOI: 10.1136/annrheumdis-2017-eular.6995

\section{AB0638 CARDIAC TRANSPLANT IN SYSTEMIC SCLEROSIS-ASSOCIATED CARDIOMYOPATHY: MONOCENTRIC EXPERIENCE OF 3 CASES}

J. Lenaerts $^{1}$, J. Lenaerts ${ }^{2}$, R. Westhovens ${ }^{2}$, W. Droogne ${ }^{3}$, A. Ciarka ${ }^{3}$, J. Van Cleemput $^{3}$, E. De Langhe ${ }^{2} .{ }^{1}$ Internal Medicine; ${ }^{2}$ Rheumatology; ${ }^{3}$ Cardiology, UZ Leuven, Leuven, Belgium

Background: Cardiac involvement in systemic sclerosis (SSc) is a frequent complication, but end-stage cardiac failure remains uncommon and represents a poor prognosis. Heart-lung and lung transplant is an established treatment option for SSc-related pulmonary disease. Due to the limited published data, no recommendations exist for cardiac transplant in the context of SSc.

Objectives: We present our monocentric experience of 3 patients with SSc who underwent cardiac transplant for SSc-related end-stage heart disease (multiple hospitalisations due to failure of medical therapy and life-threatening complications).

Results: Case 1 is a 59-year-old male with limited cutaneous SSc. Antinuclear antibody (ANA) was negative. He had vascular (digital ulcers) and cardiac (heart failure (left ventricular ejection fraction (LVEF) 20\%, NYHA class IV)) involvement, without major gastrointestinal or pulmonary involvement (no interstitial lung disease (ILD) or pulmonary arterial hypertension (PAH: assessed by right heart catheterization $(\mathrm{RHC}))$ ). He underwent a cardiac transplant at the age of 51 , after a disease duration of 6 years. Post-transplantation immunosuppressant therapy consists of tacrolimus and mycophenolic acid, initially associated with methylprednisolon, which is the standard immunosuppression protocol at our institution.

Case 2 is a 55 -year-old male with limited cutaneous SSc. ANA was positive,

\begin{tabular}{|c|c|c|c|}
\hline & Case 1 & Case 2 & Case 3 \\
\hline Sex, age & Male, 59 years & Male, 55 years & Male, 50 years \\
\hline Type of SSc & Limited & Limited & Diffuse \\
\hline Disease duration at $T x$ & 6 years & 7 years & 4 years \\
\hline $\begin{array}{l}\text { ANA } \\
\text { Pre-Tx }\end{array}$ & Negative & 1/320, speckled, no SSc-specific antibody & Negative \\
\hline NYHA class & IV & III & III \\
\hline LVEF & $20 \%$ & $40 \%$ & $40 \%$ \\
\hline RHC: $\mathrm{mPAP}$ in $\mathrm{mmHg}$ & 26 & 20 & 31 \\
\hline $\begin{array}{l}\text { RHC: PCWP in } \mathrm{mmHg} \\
\text { Post-Tx }\end{array}$ & 20 & 14 & 28 \\
\hline NYHA class & 1 & I & I \\
\hline LVEF & $60 \%$ & $55 \%$ & $60 \%$ \\
\hline RHC: $\mathrm{mPAP}$ in $\mathrm{mmHg}$ & 19 & 13 & 21 \\
\hline RHC: PCWP in $\mathrm{mmHg}$ & 12 & 8 & 12 \\
\hline
\end{tabular}

Tx: Transplant; mPAP: Mean pulmonary artery pressure; PCWP: Pulmonary capillary wedge pressure. 